\title{
INVERSES OF INFINITE SIGN REGULAR MATRICES ${ }^{1}$ \\ BY \\ C. DE BOOR, S. FRIEDLAND AND A. PINKUS
}

\begin{abstract}
Let $A$ be an infinite sign regular (sr) matrix which can be viewed as a bounded linear operator from $l_{\infty}$ to itself. It is proved here that if the range of $A$ contains the sequence $(\ldots, 1,-1,1,-1, \ldots)$, then $A$ is onto. If $A^{-1}$ exists, then $D A^{-1} D$ is also sr, where $D$ is the diagonal matrix with diagonal entries alternately 1 and -1 . In case $A$ is totally positive (tp), then $D A^{-1} D$ is also tp under additional assumptions on $A$.
\end{abstract}

0. Introduction. If the problem of spline interpolation is expressed in terms of B-splines, then the question of existence of a bounded spline interpolant to bounded data is seen to be equivalent to the question of whether a certain bounded band matrix has all bounded sequences in its range. In [5], C. A. Micchelli conjectured that there exists a unique bounded spline interpolant (of a given order and a given knot sequence) to any data sequence $\left(\tau_{i}, y_{i}\right)_{i \in \mathbf{Z}}$ in the plane, with $\left(\tau_{i}\right)$ strictly increasing and $\left(y_{i}\right)$ bounded, provided only that it is possible to interpolate the particular data sequence $\left(\tau_{i},(-1)^{i}\right)_{i \in \mathbf{Z}}$ by such a spline. There is apparently nothing special about the particular spline problem other than that it leads to a banded totally positive matrix. Therefore one of us quoted this conjecture in [3, p. 319, Problem 4] as

"A bi-infinite banded totally positive matrix $A$ is boundedly invert-

ible if and only if the linear system $A x=\left((-1)^{i}\right)$ has a bounded solution."2

Micchelli gave a simple argument for the case when $A$ is a Toeplitz matrix. Cavaretta, Dahmen, Micchelli and Smith [2] recently proved the conjecture in case $A$ is a block Toeplitz matrix. This is all the more remarkable since it is easy to see in hindsight that the conjecture is faulty even in the original context of spline interpolation. For example, interpolation by bounded broken lines with breakpoint sequence $\mathbf{Z}$ at the sequence $\tau=\mathbf{Z} \backslash\{0\}$ is possible to any bounded ordinate sequence $y$, but not uniquely so since the value of the interpolant at 0 is freely choosable. In matrix terms, this corresponds to the matrix obtained from the bi-infinite identity matrix by dropping one row. But, with the condition changed to "... has a unique bounded solution", the conjecture was proved in [1].

Received by the editors November 21, 1980.

1980 Mathematics Subject Classification. Primary 47B37; Secondary 15A09, 15A48.

Key words and phrases. Bi-infinite, infinite, matrix, total positivity, sign regularity, inverse.

${ }^{1}$ Sponsored by the United States Army under Contract No. DAAG29-80-C-0041.

${ }^{2}$ Actually, one of the editors, enlightened by [1], changed it to "a unique bounded solution" as the book went to press. 
The argument in [1] establishes that, under the given condition, $A$ " has a main diagonal", i.e., some diagonal of $A$ has the property that all finite sections of $A$ having a portion of this diagonal as their main diagonal are invertible, with their inverse bounded uniformly. $A^{-1}$ is obtained as the pointwise limit of these inverses. Thus, the argument establishes more than Micchelli's conjecture. In reaction to a presentation of these arguments, one of us suggested that there might be simpler ways to establish the conjecture directly. In particular, it should be possible, because of the checkerboard nature of inverses of totally positive matrices, to establish that $A$ is onto under the original condition, using minimal solutions of finite sections of the given linear system $A y=v$.

The present paper carries out this program in $\S 1$. As it turns out, it is possible (i) to drop any kind of structure assumption on $A$ such as bandedness, and, less surprising, (ii) the assumption of total positivity can be relaxed to sign regularity.

Having settled this matter, it then became of interest to see how much more information about the inverse of a totally positive matrix could be obtained by this approach. Specifically, assuming $A^{-1}$ to exist, and with $D$ the diagonal matrix having alternately 1 and -1 on its diagonal, could (i) the sign regularity of $D A^{-1} D$ be established, (ii) $D A^{-1} D$ or its negative be shown to be totally positive if $A$ is, (iii) $A^{-1}$ be approached by inverses of finite sections of $A$ ?

As to the third question, we show, as a simple corollary to the development in $\S 1$, that $A^{-1}$ can indeed be approached pointwise by inverses of certain submatrices of $A$ (involving consecutive columns of $A$ but not necessarily consecutive rows), provided the columns of $A$ are already in $c_{0}$ and not just bounded. We believe this assumption to be unnecessary in case $A$ is totally positive, in the sense that we believe the columns of a totally positive $l_{\infty}$-invertible matrix to be already in $c_{0}$. But we have not been able to prove this. ${ }^{3}$ In any case, while this result is far from establishing that such $A$ has a main diagonal, it does allow the conclusion that $D A^{-1} D$ or its negative is totally positive in case $A$ is.

As to the first two questions, we show in $\$ 2$ by a completely different line of reasoning that $D A^{-1} D$ must again be sign regular. From this, a surprisingly simple argument proves the total positivity of $D A^{-1} D$ in case $A$ is totally positive and infinite but not bi-infinite.

We will use the following notations and conventions.

We use lower case letters to denote elements of $\mathbf{R}^{I}$, i.e., real functions (or, sequences) on some integer interval $I$, with $v(i)$ the $i$ th entry, or value at $i$, of the sequence $v$. By $S^{-}(v)$ we mean the number of strong sign changes in the sequence $v$, i.e.,

$$
S^{-}(v):=\sup \left\{r: \text { there exist } j_{1}<\cdots<j_{r+1} \text { s.t. } v\left(j_{s}\right) v\left(j_{s+1}\right)<0\right\},
$$

while

$$
S^{+}(v):=\sup \left\{S^{-}(w): w(i)=v(i) \text { whenever } v(i) \neq 0\right\}
$$

\footnotetext{
${ }^{3}$ See Added in proof.
} 
denotes the weak sign changes of $v$. If $J$ is a subset of $I$, then $v_{J}$ denotes the restriction of $v$ to $J$ while $v_{\backslash}$ is shorthand for the restriction of $v$ to $I \backslash J$, i.e., to the complement of $J$ in $I$. If $J$ consists of just one point, $J=\{j\}$ say, then we write $y$ instead of $\backslash\{j\}$. Also, $|v|(i):=|v(i)|$, all $i$, while, if also $u \in \mathbf{R}^{I}$, then

$$
u^{*} v:=\sum_{i \in I} u(i) v(i)
$$

Correspondingly, when also $J$ is an integer interval, then $A^{*}$ denotes the transpose of the matrix $A \in \mathbf{R}^{I \times J}$ and $A_{K, L}$ denotes the restriction of $A$ to the subset $K \times L$ of $I \times J$. Such a matrix $A$ is sign regular (=:sr) provided that for each $k=1,2,3, \ldots$ all minors of $A$ of order $k$ have the same sign. If this sign is positive for all $k$, then $A$ is totally positive (=: tp). We denote the minor of $A$ obtained from rows $p<\cdots<q$ and columns $r<\cdots<s$ by

$$
A\left(\begin{array}{c}
p, \ldots, q \\
r, \ldots, s
\end{array}\right) .
$$

1. Existence of a bounded right inverse in some absolute norm. Let $J$ be a finite, infinite or bi-infinite integer interval and let $S \subseteq \mathbf{R}^{J}$ be a normed linear space of real functions on $J$, i.e., a space of sequences. We assume that the norm is absolute, i.e., for every $\varepsilon \in\{-1,1\}^{J}, s \mapsto(\varepsilon(j) s(j))$ is an isometry. We further assume that the 'unit' sequences $e^{j}, j \in J$, given by $e^{j}(i):=\delta_{i j}$, all $i, j$, form a basis for $S$, i.e., the truncation projector $P_{K}$ given by

$$
\left(P_{K} y\right)(j):= \begin{cases}y(j), & j \in K, \\ 0, & \text { otherwise, }\end{cases}
$$

converges strongly to 1 as the finite interval $K$ approaches $J$. Then the continuous dual $S^{*}$ of $S$ can be identified with the sequence space

$$
\left\{f \in \mathbf{R}^{J}:\|f\|^{*}:=\sup _{s \in S} f^{*} s /\|s\|<\infty\right\},
$$

and the norm on $S^{*}$ is again absolute. In particular, $|f|^{*}|s| \leqslant\|f\|^{*}\|s\|$, all $f \in S^{*}$, $s \in S$.

Let $A \in \mathbf{R}^{I \times J}$ for some finite, infinite or bi-infinite integer intervals $I$ and $J$ and assume that $A(i, \cdot) \in S$, all $i$. Then we can identify $A$ with the linear map

$$
S^{*} \rightarrow \mathbf{R}^{I}: f \mapsto A f .
$$

We are interested in understanding the range of this map under the assumption that $A$ is sr.

THEOREM 1. Let I, J be finite, infinite or bi-infinite integer intervals, and let $S \subseteq \mathbf{R}^{J}$ be a normed linear space with absolute norm and with $\left(e^{j}\right)_{j \in J}$ as a basis. If $A \in \mathbf{R}^{I \times J}$ is $s r$, has its rows in $S$, and carries some $x \in S^{*}$ to the strictly alternating sequence $u:=A x$, then the range of $A$ contains the Banach space

$$
S_{u}:=\left\{v \in \mathbf{R}^{I}:\|v\|_{u}:=\sup _{i \in I}|v(i) / u(i)|<\infty\right\} .
$$

More explicitly, for every $v \in S_{u}$ there exists $y_{v} \in S^{*}$ so that $A y_{v}=v$ and $\left\|y_{v}\right\|^{*} \leqslant$ $\|v\|_{u}\|x\|^{*}$. 
Proof. We first consider the case that $I$ is finite. Since $S^{-}(u)=|I|-1$, we claim that $A$ has full rank $|I|$ and is therefore onto. Indeed, by induction, we may assume that $A$ has rank at least $|I|-1$. If now rank $A=|I|-1$, then there would be, up to scalar multiples, a unique $z \in \mathbf{R}^{I} \backslash\{0\}$ for which $z^{*} A=0$. Then the sign regularity of $A$ would imply that $z$ must alternate, i.e., $z(i) z(i+1) \leqslant 0$, all $i$. Therefore $0=z^{*} A x=z^{*} u$, and strict alternation of $u$ would then imply that $z=0$, a contradiction.

It follows that every $v \in \mathbf{R}^{I}$ gives rise to a linear functional $F_{v}$ defined on the finite-dimensional linear space $R:=\operatorname{span}(A(i, \cdot))_{i \in I}$ by the rule

$$
\sum_{i \in I} \alpha_{i} A(i, \cdot) \mapsto \alpha^{*} v
$$

In view of the Hahn-Banach Theorem, we can therefore conclude the existence of $y_{v} \in S^{*}$ with $A y_{v}=v$ and $\left\|y_{v}\right\|^{*} \leqslant\|v\|_{u}\|x\|^{*}$ once we prove that $\left\|F_{v}\right\| \leqslant$ $\|v\|_{u}\|x\|^{*}$.

It is sufficient to consider only finite $J$, for an infinite $J$ can always be approached by finite intervals $K$, and

$$
A(i, \cdot)=\lim _{K \rightarrow J} P_{K} A(i, \cdot), \quad \text { all } i,
$$

by assumption. Therefore, for all sufficiently large intervals $K$, the rule $\Sigma \alpha_{i} P_{K} A(i, \cdot)$ $\mapsto \alpha^{*} v$ defines a linear functional $F_{v}^{K}$ on $R^{K}:=P_{K}[R]$ and $\lim _{K \rightarrow J}\left\|F_{v}^{K}\right\|=\left\|F_{v}\right\|$.

Next, we establish the following auxiliary

Claim. For any $s \in S$ with $\|s\|=1$, there exists $K$ in $J$ so that $A_{I, K}$ is invertible and, for all $v \in \mathbf{R}^{I},\left|\left(A_{I, K}\right)^{-1} v\right|^{*}|s| \leqslant\|v\|_{u}\|x\|^{*}$.

For its proof (which incorporates a suggestion by Rong-qing Jia for the improvement of an earlier argument), let $w$ be an element of minimal support from the set

$$
C:=\left\{y \in S^{*}: A y=u,|y|^{*}|s| \leqslant|x|^{*}|s|\right\} .
$$

Such a $w$ exists since $J$ is finite and since $x \in C$, hence $C$ is not empty.

We claim that $K:=\operatorname{supp} w$ contains no more than $|I|$ points and prove this by an argument familiar from linear programming. Indeed, if $K$ were to contain a set $L$ with $|L|=|I|+1$, then we could find $z \in S^{*} \backslash\{0\}$ with $A z=0$ and supp $z \subseteq L$. From this, we could produce an element $w^{\varepsilon}:=w-\varepsilon z \in C$ with $\left|\operatorname{supp} w^{\varepsilon}\right|<$ $|\operatorname{supp} w|$ as follows. First, $A w^{\varepsilon}=u$ for all $\varepsilon$. Further, as long as

$$
w(j)[w(j)-\varepsilon z(j)] \geqslant 0, \quad \text { for all } j \in L,
$$

i.e., for all $\varepsilon$ in an open interval containing 0 (since $L \subseteq \operatorname{supp} w$ ), we have

$$
\left|w^{\varepsilon}\right|^{*}|s|=\sum|w(j)-\varepsilon z(j)||s(j)|=|w|^{*}|s|-\varepsilon \sigma
$$

with $\sigma:=\sum \operatorname{sign}[w(j)] z(j)|s(j)|$. Therefore, assuming without loss of generality that $\sigma \geqslant 0$ with $w(j) z(j)>0$ for some $j$, the choice $\varepsilon=\min \{w(j) / z(j): w(j) z(j)$ $>0\}$ would work. 
With this, $|K| \leqslant|I|$ and then, since $A_{I, K} w_{K}=u_{I}$, our earlier argument implies that $A_{I, K}$ is invertible and, in particular, $|K|=|I|$. But now,

$$
\begin{aligned}
\left|\left(A_{I, K}\right)^{-1} v\right|^{*}|s| & =\sum_{k \in K}\left|\sum_{i \in I}\left(A_{I, K}\right)^{-1}(k, i) v(i)\right||s(k)| \\
& \leqslant\|v\|_{u} \sum_{k \in K} \sum_{i \in I}\left|\left(A_{I, K}\right)^{-1}(k, i)\right||u(i)||s(k)|
\end{aligned}
$$

and

$$
\begin{aligned}
\sum_{k \in K} \sum_{i \in I}\left|\left(A_{I, K}\right)^{-1}(k, i)\right||u(i)||s(k)| & =\sum_{k \in K}\left|\sum_{i \in I}\left(A_{I, K}\right)^{-1}(k, i) u(i)\right||s(k)| \\
& =|w|^{*}|s| \leqslant|x|^{*}|s| \leqslant\|x\|^{*}
\end{aligned}
$$

by the sign regularity of $A$ (which gives that $\left(A_{I, K}\right)^{-1}$ must be checkerboard), the alternation of $u$, and the fact that $w \in C$. This proves the claim.

In particular, choosing now $s$ as an extremal for $F_{v}$, i.e., so that $s \in R,\|s\|=1$, $F_{v} s=\left\|F_{v}\right\|$, we conclude from the claim that

$$
\left\|F_{v}\right\|=F_{v} s=\left(\left(A_{I, K}\right)^{-1} v\right){ }^{*} s \leqslant\|v\|_{u}\|x\|^{*} .
$$

This establishes the existence of $y_{v} \in S^{*}$ with $A y_{v}=v$ and $\left\|y_{v}\right\|^{*} \leqslant\|v\|_{u}\|x\|^{*}$ for finite $I$. From this, we obtain the result for nonfinite $I$ by considering all finite integer intervals $L$ contained in $I$. For each such $L$, we can find $y_{v}^{L} \in S^{*}$ with $A y_{v}^{L}=v_{L}$ and $\left\|y_{v}^{L}\right\|^{*} \leqslant\left\|v_{L}\right\|_{u}\|x\|^{*} \leqslant\|v\|_{u}\|x\|^{*}$. Therefore, for some increasing sequence $(L)$ converging to $I$, the corresponding sequence $\left(y_{v}^{L}\right)$ converges weak* to some $y_{v} \in S^{*}$. But then also $\left\|y_{v}\right\|^{*} \leqslant\|v\|_{u}\|x\|^{*}$ and

$$
\begin{aligned}
\left(A y_{v}\right)(i)=y_{v}^{*} A(i, \cdot) & =\lim _{L \rightarrow I}\left(y_{v}^{L}\right)^{*} A_{L, J}(i, \cdot) \\
& =\lim _{L \rightarrow I}\left\{\begin{array}{ll}
v(i), & i \in L \\
\text { undefined, } & i \notin L
\end{array}\right\}=v(i) .
\end{aligned}
$$

As a special case, consider the sr matrix $A \in \mathbf{R}^{I \times J}$ to carry $l_{\infty}(J)$ to $l_{\infty}(I)$. Its rows must then be in $l_{1}(J)$, a sequence space with absolute norm and $\left(e^{j}\right)$ as a basis. At the same time, $S_{u}=l_{\infty}(I)$ provided $u$ alternates uniformly, i.e., $u(i) u(i+1)<0$, all $i$, and inf $|u(i)|>0$. We therefore have the following

COROLlARY 1. If I and $J$ are finite, infinite or bi-infinite integer intervals and the sr matrix $A \in \mathbf{R}^{I \times J}$ carries $l_{\infty}(J)$ to $l_{\infty}(I)$ in such a way that, for some $x \in l_{\infty}(J)$, $u:=A x$ uniformly alternates, then $A$ is onto.

REMARK. This corollary establishes the full generalization of Micchelli's conjecture. The theorem even shows that the solution $y$ of $A y=v$ may be chosen bounded in terms of $v$, i.e., $\|y\|_{\infty} \leqslant k\|v\|_{\infty}$ with $k:=\sup _{i, j}|x(i) / u(j)|$ independent of $v$, and also demonstrates all this without the assumption that $A$ is 1-1.

As a second special case, consider the sr matrix $A \in \mathbf{R}^{I \times J}$ to have all its rows in $S=c_{0}(J)$, another sequence space with absolute norm and $\left(e^{j}\right)$ as a basis. Then $\|y\|^{*}=\|y\|_{1}:=\Sigma|y(j)|=|y|^{*}|s|$ with $s(j)=1$, all $j$. The claim established during the proof of Theorem 1 therefore assures us that we can choose, for each 
finite interval $L$ in $I$, a subset $K$ of $J$ with $|K|=|L|$ so that

$$
\text { for all } v \in \mathbf{R}^{I}, \quad\left\|\left(A_{L, K}\right)^{-1} v_{L}\right\|_{1} \leqslant\|v \cdot\|_{u}\|x\|_{1} .
$$

Next we extend $\left(A_{L, K}\right)^{-1}$ to $C^{L} \in R^{J \times I}$ by taking its values to be zero off $K \times L$. For each $i \in I, e^{i}$ is in $S_{u}$. The above argument therefore shows that $\left\|C^{L} e^{i}\right\|_{1} \leqslant$ $\|x\|_{1}\left\|e^{i}\right\|_{u}=\|x\|_{1} /|u(i)|$. We can therefore choose a sequence $(L)$ and a corresponding sequence $(K)$ so that, for each $i, C^{L} e^{i}$ converges weak* to some $y^{i} \in l_{1}(J)$. This means that for all $a \in c_{0}(J), \lim _{L \rightarrow I} a^{*} C^{L} e^{i}=a^{*} y^{i}$ and so in particular

$$
\text { for all } r, \quad\left(A y^{i}\right)(r)=\lim _{L \rightarrow I} A\left(C^{L} e^{i}\right)(r)=\lim _{L \rightarrow I}\left\{\begin{array}{ll}
e^{i}(r), & i \in L \\
0, & i \notin L
\end{array}\right\}=e^{i}(r) \text {. }
$$

This shows that the matrix $C$ given by $C(j, i):=y^{i}(j)$, all $(j, i) \in J \times I$, is the pointwise limit of the sequence $\left(C^{L}\right)$. It is a right inverse of $A$ and it satisfies

$$
\|C(\cdot, i)\|_{1} \leqslant\|x\|_{1} /|u(i)|, \quad \text { all } i \text {. }
$$

This proves

COROllaRY 2. Let $I$ and $J$ be finite, infinite or bi-infinite integer intervals. If $A \in \mathbf{R}^{I \times J}$ is sr, has its rows in $c_{0}(J)$, and carries some $x \in l_{1}(J)$ to the strictly alternating sequence $u:=A x$, then there exist a sequence $(L)$ of index intervals converging to $I$ and a corresponding sequence $(K)$ of index sets so that $\left(A_{L, K}\right)^{-1}$ exists and converges pointwise to a matrix $C \in \mathbf{R}^{J \times I}$ which carries $S_{u}$ to $l_{1}(I)$ and satisfies $A C=1$ (as maps, hence as matrices).

2. The inverse of a sr matrix. In this section, we assume that the sr matrix $A \in \mathbf{R}^{I \times J}$ is also 1-1, as a map on $l_{\infty}$, in addition to having a uniformly alternating sequence in its range. We then know that $A$ is 1-1 and onto, hence invertible, with $A^{-1}$ again (representable as) a matrix, from $\mathbf{R}^{J \times I}$, which carries $l_{\infty}(I)$ onto $l_{\infty}(J)$.

Let now $D^{I} \in \mathbf{R}^{I \times I}$ be the diagonal matrix whose diagonal entries are alternately 1 and -1 . Specifically,

$$
\left(D^{I} y\right)(i)=(-)^{i} y(i), \quad \text { all } i \in I, \text { all } y \in \mathbf{R}^{I}
$$

if $I$ is an interval (as we assume). It is well known that, for finite $I$ and $J$, the matrix $D^{J} A^{-1} D^{I}$ is again sr. In addition, if $A$ is tp, then $D^{J} A^{-1} D^{I}$ or its negative is also tp. We prove the first statement for arbitrary $I$ and $J$, and prove the second statement under the additional assumption that the columns of $A$ are in $c_{0}$ or else that $I$ equals $J$ and is not $b i$-infinite, i.e., has a first or last entry.

Proposition 1. If $A \in \mathbf{R}^{I \times J}$ maps $l_{\infty}(J)$ to $l_{\infty}(I)$ and is $1-1$ and onto, and maps $c_{0}(J)$ to $c_{0}(I)$, then $A$ tp implies that $D^{J} A^{-1} D^{I}$ or its negative is tp.

Proof. We know from Corollary 2 to Theorem 1 that, under the given assumptions, $A^{-1}$ is the pointwise limit of certain matrices $\left(C^{L}\right)^{*}$ as the index interval $L$ converges to $I$. The matrix $C^{L}$ equals $\left(A_{K, L}\right)^{-1}=\left(A_{L, K}{ }^{*}\right)^{-1}$ on $K \times L$ and vanishes off $K \times L$. Here $L$ is an interval, but $K$ is only an index set, $K=\left\{k_{1}, \ldots, k_{r}\right\}$, say, with $k_{1}<\cdots<k_{r}$. For such $K$, we define the diagonal matrix $D^{K}$ by

$$
\left(D^{K} y\right)\left(k_{i}\right)=(-)^{i} y\left(k_{i}\right), \quad i=1, \ldots, r \text {, all } y \in \mathbf{R}^{K} \text {. }
$$


Then $D^{L}\left(A_{K, L}\right)^{-1} D^{K}$ is tp since $A_{K, L}$ is. Now every $i$ in $I$ must eventually be in all $K$ 's since in the contrary case the $i$ th row of $\left(C^{L}\right)^{*}$ would be zero for infinitely many $L$, hence $A^{-1}(i, \cdot)=0$, which is nonsense. Thus, for any finite intervals $M$ and $N,\left(A^{-1}\right)_{M, N}$ is the pointwise limit of $\left(A_{K, L}\right)_{M, N}^{-1}$ as $L \rightarrow I$, with $\varepsilon_{M, N} D^{M}\left(A_{K, L}\right)_{M, N}^{-1} D^{N}$ tp for some $\varepsilon_{M, N} \in\{-1,1\}$. This implies that $\varepsilon_{M, N}$ is independent of $M$ and $N$, and so $D^{M}\left(A^{-1}\right)_{M, N} D^{N}$ or its negative is tp. But since $M$ and $N$ are arbitrary finite intervals, this implies that $D^{J} A^{-1} D^{I}$ or its negative is tp.

We believe the assumption that $A$ map $c_{0}(J)$ to $c_{0}(I)$ to be unnecessary for the conclusion that $D^{J} A^{-1} D^{I}$ or its negative is tp. More precisely, we conjecture ${ }^{4}$ that a $l_{\infty}$-invertible tp matrix $A \in \mathbf{R}^{I \times J}$ must map $c_{0}(J)$ to $c_{0}(I)$. Without this assumption, we have no way of approximating $A^{-1}$ by inverses of certain finite submatrices of $A$, and will have to prove by some other means that $D^{J} A^{-1} D^{I}$ is sr in case $A$ is sr.

THEOREM 2. Let $I, J$ be finite, infinite or bi-infinite intervals. If $A \in \mathbf{R}^{I \times J}$ is sr and invertible as a map from $l_{\infty}(J)$ to $l_{\infty}(I)$, then $D^{J} A^{-1} D^{I}$ is also sr.

Proof. In outline, the proof is as follows. By well-known results, it is sufficient to prove that $D^{J} A^{-1} D^{I}$ is variation diminishing, i.e.,

$$
S^{-}\left(D^{J} A^{-1} D^{I} z\right) \leqslant S^{-}(z), \text { all } z,
$$

and this is equivalent to the assertion that $u=A x$ implies $S^{-}\left(D^{J} x\right) \leqslant S^{-}\left(D^{I} u\right)$. This, in turn, follows by a smoothing argument from the assertion that

$$
u=A x \text { and } u, x \text { nowhere zero implies } S^{+}\left(D^{J} x\right) \leqslant S^{+}\left(D^{I} u\right),
$$

and, finally, this last statement follows, as we will show, from the assertion that

$$
u=A x \text { and } u \text { nowhere zero implies } \quad x \text { vanishes at most } S^{+}\left(D^{I} u\right) \text { times. }
$$

We begin the detailed argument with a proof of this last assertion and for this start with the following

LEMMA 1. If $B \in \mathbf{R}^{I \times J}$ is 1-1, then $B_{I, J \backslash j}$ is still 1-1 but not onto.

Proof. Since $B$ is $1-1$, the sequence $B(\cdot, j)$ cannot be in the range of $B_{I, J \backslash j}$, hence $B_{I, J \backslash j}$ is not onto. On the other hand, if $B_{I, J \backslash j} x=B_{I, J \backslash j} y$, then, extending $x$ and $y$ to all of $J$ by setting them equal to 0 at $j$ gives $B x=B y$, hence $x=y$.

COROLlary. If $u:=$ Ax uniformly alternates, then $x$ vanishes nowhere.

Proof. If $x$ were to vanish at $j$, then the sr matrix $A_{I, J \backslash j}$ would carry the bounded sequence $x_{\backslash j}$ to the uniformly alternating sequence $u$ and Corollary 1 to Theorem 1 would give that $A_{I, J \backslash j}$ is onto, while $A$ is 1-1 by assumption, hence $A_{I, J \backslash j}$ is not onto by the lemma.

Next, we strengthen this corollary as follows.

Proposition 2. Suppose $u=A x$ satisfies $\inf _{i}|u(i)|>0$ and $S^{+}\left(D^{I} u\right)=k$, while $x_{L}=0$ for some $L$ with $|L|=k$. Let $K:=\{i \in I: u(i) u(i+1)>0\}$. Then, the matrix $C:=A_{\backslash K, \backslash L}$ is again sr, 1-1 and onto.

\footnotetext{
${ }^{4}$ See Added in proof.
} 
Proof. Since $u$ never vanishes and $S^{+}\left(D^{I} u\right)=k$, therefore $|K|=k$ and the subsequence $u_{\backslash K}$ of $u$ alternates uniformly. In addition, $C x_{\backslash L}=u_{\backslash K}$ and $C$ is sr. Therefore $C$ is onto by Corollary 1 to Theorem 1 .

To prove that $C$ is $1-1$, let $C z=0$ for some $z \in l_{\infty}(V)$, and extend $z$ to $\tilde{z} \in l_{\infty}(J)$ by $\tilde{z}_{L}=0$. Set $y:=A \tilde{z}$. Then $y_{\backslash K}=0$.

Since $C$ is onto, we can find, for each $j \in L$, a bounded solution $x^{j}$ to the problem $x_{L}^{j}=0, A x^{j}=A e^{j}$ off $K$, with $e^{j}(i):=\delta_{i j}$, all $i, j$, as before. Set

$$
F: \mathbf{R}^{L} \rightarrow l_{\infty}(I): \alpha \mapsto \sum_{j \in L} \alpha_{j}\left(e^{j}-x^{j}\right) .
$$

Then $F \alpha=\alpha$ on $L$ while $A F \alpha=\sum_{j \in L} \alpha_{j}\left(A e^{j}-A x^{j}\right)$ vanishes off $K$. Therefore, $A F \alpha=0$ on $K$ implies $A F \alpha=0$ and so, $A$ being 1-1, we get $F \alpha=0$ and, in particular, $\alpha=(F \alpha)_{L}=0$. This shows that $\mathbf{R}^{L} \rightarrow \mathbf{R}^{K}: \alpha \mapsto(A F \boldsymbol{\alpha})_{K}$ is 1-1, hence onto since $|L|=|K|$.

It follows that we can choose $\alpha$ so that $A F \alpha=y$ on $K$. But then $z^{\prime}:=\tilde{z}-F \alpha$ satisfies

$$
A z^{\prime}=y-A F \alpha=\left\{\begin{array}{ll}
y-y & \text { on } K \\
0-0 & \text { off } K
\end{array}\right\}=0
$$

and so, $A$ being 1-1, we have $z^{\prime}=0$; therefore $0=z_{L}^{\prime}=0-\alpha$, i.e., $\alpha=0$ and so, finally, $z=\tilde{z}_{\backslash L}=(\tilde{z}-F \boldsymbol{\alpha})_{\backslash L}=z_{\backslash L}^{\prime}=0$.

REMARK. The argument just given shows the following general fact: If the linear map $B$ is $1-1$ and can be partitioned as

$$
B=\left[\begin{array}{ll}
B_{11} & B_{12} \\
B_{21} & B_{22}
\end{array}\right]
$$

in such a way that $B_{11}$ is onto while $B_{22}$ is square of finite order $k$, then $B_{11}$ is also $1-1$.

COROllaRY. If $u=A x \in l_{\infty}(I)$ with $\inf _{i}|u(i)|>0$ and $S^{+}\left(D^{I} u\right)=k$, then $x$ has at most $k$ zero entries.

Proof. Let $x_{L}=0$ for some $L$ with $|L|=k$. Setting again

$$
K:=\{i \in I: u(i) u(i+1)>0\},
$$

we know from Proposition 2 that $C:=A_{\backslash K, \backslash L}$ is $1-1$, while it obviously carries $x_{\backslash L}$ to the uniformly alternating sequence $u_{\backslash K}$ and is sr. Therefore, by the corollary to Lemma 1, $x$ does not vanish off $L$.

Lemma 2. If $u=A x$ with $\inf _{i}|u(i)|>0$ and $S^{+}\left(D^{I} u\right)=k$, then $S^{+}\left(D^{J} x\right) \leqslant k$.

Proof. We first show that we may assume that $x$ vanishes nowhere. For, if this is not the case, then we replace each zero entry of $x$ by $\varepsilon$ or $-\varepsilon$ in such a way that the resulting sequence $x^{\varepsilon}$ satisfies $S^{+}\left(D^{J} x^{\varepsilon}\right)=S^{+}\left(D^{J} x\right)$. This changes $u=A x$ to $u^{\varepsilon}:=A x^{\varepsilon}=u+v$ with $\|v\|_{\infty} \leqslant\|A\||\varepsilon|$. But since $\inf _{i}|u(i)|>0$, we can choose $\varepsilon>0$ so small that again $\inf _{i}\left|u^{\varepsilon}(i)\right|>0$, while $S^{+}\left(D^{I} u^{\varepsilon}\right)=S^{+}\left(D^{I} u\right)$.

Next we produce a sr 1-1 onto matrix $C$ and a sequence $z$ with as many zeros as $D^{J} x$ has sign changes and with $C z=u$. For this, consider the matrix $E_{i}(a)$ which 
differs from the identity only in that it has an $a$ in position $(i+1, i)$. This matrix is tp for nonnegative $a$ and carries the sequence $z$ to itself except that the $(i+1)$ st entry is changed to $z(i+1)+a z(i)$. Consequently, $E_{i}(a)$ is invertible, with $E_{i}(-a)$ its inverse.

Now let $r_{i}:=x(i+1) / x(i)$. If $x(i) x(i+1)>0$, then $r_{i}>0$ and $y:=E_{i}\left(-r_{i}\right) x$ equals $x$ except for a zero in entry $i+1$. Hence, if $i_{1}<\cdots<i_{n}$ are all in $K:=\{i \in I: x(i) x(i+1)>0\}$, then the matrix $B:=E_{i_{1}}\left(-r_{i_{1}}\right) \cdots E_{i_{n}}\left(-r_{i_{n}}\right)$ carries $x$ to a sequence which vanishes at $i_{1}+1, \ldots, i_{n}+1$, while

$$
B^{-1}=E_{i_{n}}\left(r_{i_{n}}\right) \cdots E_{i_{1}}\left(r_{i_{1}}\right)
$$

is tp, 1-1 and onto; hence $A B^{-1}$ is again sr, $1-1$ and onto. Since $A B^{-1}(B x)=u$, we now conclude from the corollary to Proposition 2 that $n \leqslant k$. This proves the lemma in view of the fact that $S^{+}\left(D^{J} x\right)=|K|$, since $x$ vanishes nowhere.

Lemma 3. If $A x=u$, then $S^{-}\left(D^{J} x\right) \leqslant S^{-}\left(D^{I} u\right)$.

Proof. There is nothing to prove unless $S^{-}\left(D^{I} u\right)<\infty$. In that case, we choose $\operatorname{sign}[u(i)] \in\{-1,1\}$ in such a way that $S^{+}\left(D^{I}(\operatorname{sign}[u(\cdot)])\right)=S^{-}\left(D^{I} u\right)$ and then set

$$
u^{\varepsilon}(i):= \begin{cases}\varepsilon \operatorname{sign}[u(i)] & \text { if }|u(i)|<\varepsilon, \\ u(i) & \text { otherwise. }\end{cases}
$$

Then $S^{+}\left(D^{I} u^{\varepsilon}\right)=S^{-}\left(D^{I} u\right)$ and so, using the boundedness of $A^{-1}$,

$$
S^{-}\left(D^{J} x\right) \leqslant \underline{\lim } S^{+}\left(D^{J}\left(A^{-1} u^{\varepsilon}\right)\right) \leqslant S^{+}\left(D^{I} u^{\varepsilon}\right)=S^{-}\left(D^{I} u\right),
$$

by Lemma 2 .

With this, the proof of Theorem 2 is apparent. For we now conclude from Lemma 3 that $S^{-}\left(D^{J} A^{-1} D^{I} z\right) \leqslant S^{-}(z)$, all $z$, and therefore every finite submatrix of $D^{J} A^{-1} D^{I}$ is variation-diminishing. Hence, by Karlin [4, p. 222], $D^{J} A^{-1} D^{I}$ is sr.

Corollary. If $I=J$ is only infinite (and not bi-infinite), then $A$ tp implies $D A^{-1} D$ $t p$.

Proof. Assume without loss that $I=\{1,2,3, \ldots\}$ and consider the matrix $B \in$ $\mathbf{R}^{K \times K}$ with $K:=\{0\} \cup I$ and

$$
B:=\left[\begin{array}{ll}
1 & 0 \\
0 & A
\end{array}\right]
$$

Since $A$ is tp and invertible, so is $B$, with

$$
B^{-1}=\left[\begin{array}{cc}
1 & 0 \\
0 & A^{-1}
\end{array}\right] \text {. }
$$

Further, both $D^{K} B^{-1} D^{K}$ and $D^{I} A^{-1} D^{I}$ are sr, by Theorem 2 . For $k=0,1,2, \ldots$, let $\varepsilon_{k}$ denote the common sign of the $k \times k$ minors of $D^{K} B^{-1} D^{K}$, hence of $D^{I} A^{-1} D^{I}$. Then, for any $k$,

$$
D^{K} B^{-1} D^{K}\left(\begin{array}{l}
0, \ldots, k \\
0, \ldots, k
\end{array}\right)=D^{I} A^{-1} D^{I}\left(\begin{array}{l}
1, \ldots, k \\
1, \ldots, k
\end{array}\right)
$$

and, since these minors are nonzero, we conclude that $\varepsilon_{k+1}=\varepsilon_{k}$, all $k$, therefore $\varepsilon_{k}=\varepsilon_{0}=1$, all $k$. 
Added IN Proof. In March, 1981, Rong-qing Jia communicated to us a proof of our conjecture that a sr matrix carrying $l_{\infty}$ to $l_{\infty}$ and invertible must carry $c_{0}$ to $c_{0}$. This opened the way to an argument (based on Corollary 2 to Theorem 1) showing that an invertible tp matrix has one (and only one) main diagonal. These results and others are the subject of the paper on "Structure of invertible (bi)infinite totally positive matrices", by C. de Boor, R.-q. Jia and A. Pinkus, MRC TSR \#2311 (1981), to appear in Linear Algebra Applications.

\section{REFERENCES}

1. C. de Boor, The inverse of a totally positive bi-infinite band matrix, MRC TSR 2155 (1980); Trans. Amer. Math. Soc. 274 (1982), 45-58.

2. A. Cavaretta, W. Dahmen, C. A. Micchelli and P. Smith, On the solvability of certain systems of linear difference equations, RC 8329, IBM Research Report, 1980.

3. R. DeVore and K. Scherer, eds., Quantitative approximation, Academic Press, New York, 1980.

4. S. Karlin, Total positivity, Stanford Univ. Press, Stanford, 1968.

5. C. A. Micchelli, Infinite spline interpolation, Approximation in Theorie und Praxis. Ein Symposiumsbericht (G. Meinardus, ed.), Bibliographisches Institut, Mannheim, 1979, pp. 209-238.

Mathematics Research Center, University of Wisconsin, Madison, Wisconsin 53706

InStitute of Mathematics, Hebrew University of JeRUSAlEm, Jerusalem, ISRAEL

Department of Mathematics, The Technion, Haifa, IsRael 